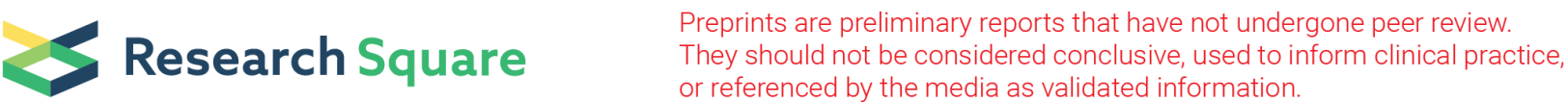

\section{Structural basis for the mechanisms of human presequence protease conformational switch and substrate recognition}

\section{Wenguang Liang}

Ben-May Department for Cancer Research, The University of Chicago

\section{Juwina wijaya}

Department of Chemistry and Biochemistry, University of California Los Angeles

Hui Wei

New York Structural Biology Center

Alex Noble

New York Structural Biology Center https://orcid.org/0000-0001-8634-2279

Swansea Mo

$U$ of Chicago

David Lee

UCSD https://orcid.org/0000-0001-7232-0968

Jordan Mancl

$U$ of Chicago

John Lin King

UCSF

Man Pan

University of Chicago

chang Liu

$\mathrm{U}$ of Chicago

Carla Koehler

UCLA

\section{Minglei Zhao}

University of Chicago https://orcid.org/0000-0001-5832-6060

\section{Clinton Potter}

Simons Electron Microscopy Center

\section{Bridget Carragher}

The National Resource for Automated Molecular Microscopy, Simons Electron Microscopy Center, New York Structural Biology Center, New York, NY https://orcid.org/0000-0002-0624-5020

\section{Sheng Li}

University of California at San Diego https://orcid.org/0000-0002-9073-6809 


\section{Wei-Jen Tang ( $\nabla$ wtang@uchicago.edu )}

University of Chicago https://orcid.org/0000-0002-8267-8995

\section{Article}

Keywords: Presequence protease, amyloid peptide, mitochondrial proteostasis, cryoEM, crystallography, hydrogen-deuterium exchange, Alzheimer's disease, integrative structural biology

Posted Date: August 31st, 2020

DOI: https://doi.org/10.21203/rs.3.rs-63850/v1

License: (c) (1) This work is licensed under a Creative Commons Attribution 4.0 International License. Read Full License

Version of Record: A version of this preprint was published at Nature Communications on April 5th, 2022. See the published version at https://doi.org/10.1038/s41467-022-29322-4. 


\section{Abstract}

Presequence protease (PreP), a $117 \mathrm{kDa}$ mitochondrial M16C metalloprotease vital for mitochondrial proteostasis, degrades presequence peptides cleaved off from nuclear-encoded proteins and other aggregation-prone peptides, such as amyloid $\beta(A \beta)$. PreP structures have only been determined in a closed conformation; thus, the mechanisms of substrate binding and selectivity remain elusive. Here, we leverage advanced vitrification techniques to overcome the preferential denaturation of one of two 55 $\mathrm{kDa}$ homologous domains of PreP caused by air-water interface adsorption, and thereby elucidate cryoEM structures of three apo-PreP open states along with $A \beta$ - and citrate synthase presequence-bound PreP at $3.3 \AA$ A-4.6 $\AA$ resolution. Together with integrative biophysical and pharmacological approaches, these structures reveal the key stages of the PreP catalytic cycle and how the binding of substrates or PreP inhibitor drives the rigid body motion of the protein for substrate binding and catalysis. Together, our studies provide key mechanistic insights into M16C metalloproteases for future therapeutic innovations.

\section{Introduction}

Mitochondria are vital to cellular metabolism, homeostasis, and stress responses ${ }^{1,2}$; their defects are linked to a plethora of neurodegenerative diseases ${ }^{3}$. Assembly of mitochondria requires the coordinated action of protein import into mitochondria, coupled with processing and proteolysis pathways ${ }^{4,5}$. The majority of imported proteins contain a presequence (also known as a mitochondrial targeting sequence) at the $\mathrm{N}$-terminus ${ }^{4}$. Upon entering into mitochondria, presequences are cleaved off by mitochondrial processing peptidase and in some cases further cleaved by mitochondrial intermediate proteases ${ }^{4,5}$. The cleaved presequence is then degraded by presequence protease (PreP). If left to accumulate, presequences, rich in hydrophobic and positively charged residues, are highly toxic to mitochondria 4,5 . PreP is a ubiquitously expressed, $117 \mathrm{kDa} \mathrm{M} 16 \mathrm{C}$ clan zinc metalloprotease that localizes to the mitochondria matrix and cleaves presequence peptides into non-toxic pieces ${ }^{4,6,7}$. PreP also degrades amyloid $\beta(A \beta)$, the cleavage products of the amyloid precursor protein (APP) that is linked to the progression of Alzheimer's disease and is imported into mitochondria, particularly synaptic mitochondria ${ }^{6-9}$. Pitrm 1 (encodes for PreP) knockout mice display embryonic lethality ${ }^{10}$. Furthermore, genetic defects in PreP are linked with human neurological disorders, e.g., cognitive disability/impairment and cerebellar atrophy ${ }^{10,11}$.

Proteomes are maintained in a healthy state by four main processes, autophagy, chaperones, ubiquitination/proteasomal degradation, and a cohort of proteases that degrade potentially cytotoxic peptides $^{12,13}$. Aggregates of such peptides include amyloidogenic species that are highly cytotoxic and are associated with human neurodegenerative diseases, e.g., Alzheimer's and Parkinson's disease, and non-neuropathic systemic amyloidoses ${ }^{14-16}$. $A \beta$ is a key initiating factor in Alzheimer's disease and its accumulation is caused by the imbalance between $A b$ production and clearance ${ }^{16}$. $A \beta$ is the proteolytic product of the amyloid precursor protein (APP), which belongs to a small gene family including APP-like proteins that have shared functions in CNS development, synapse formation, brain injury and 
neuroprotection ${ }^{17}$. Only through the processing of APP, a recently evolved gene within the APP family, can generate $A \beta$, and no dedicated protease has evolved for $A \beta$ clearance ${ }^{17}$. Only a handful of proteases out of 569 human proteases can effectively degrade monomeric $A \beta^{6,18,19}$. A $\beta$-degrading proteases have a broad subcellular distribution, e.g., extracellular milieu (insulin degrading enzyme (IDE), matrix metalloprotease 2 (MMP2), MMP9); mitochondria (PreP), intracellular vesicles (IDE), lysosomes (cathepsin B), the plasma membrane (neprilysin (NEP) and endothelin converting enzyme 1 and 2 (ECE1/2), plasmin, IDE), and the cytosol (IDE, APEH), allowing for better control of Ab levels at all intraand extra-cellular locations where $\mathrm{Ab}$ has been detected.

The formation of aggregates of amyloid peptides such as Ab is at least a two-step process; the first is a slow, stochastic, and reversible nucleation to form small amyloid peptide seeds followed by the elongation of seeds into large amyloid fibrils, which is faster and mostly irreversible ${ }^{20,21}$. Monomeric amyloid peptide fuels the forward progression of both steps; thus, Ab-degrading proteases recognize and degrade monomeric amyloid peptides to prevent the formation of amyloid fibrils ${ }^{18}$. Of the A proteases, PreP belongs to the chamber-containing proteases (crypt-containing peptidases, or cryptidases) that use a sizable catalytic chamber to engulf, unfold, and degrade their substrates ${ }^{22}$. Other cryptidases include IDE (M16A clan) and M13 metalloproteases, e.g., NEP and ECE1/2 22. Ab-degrading proteases also can selectively degrade other amyloid peptides which are highly diverse in sequence and structure. This poses a major challenge for how proteases selectively degrade amyloid peptides over nonamyloidogenic peptides.

Crystallographic analyses reveal that PreP and the related M16C metalloproteases are composed of $55 \mathrm{kDa}$ homologous $\mathrm{N}$ - and C-terminal domains (PreP-N and PreP-C, respectively), which are connected by an extended helical hairpin ${ }^{6,7}$. PreP-N and PreP-C, in the closed state of PreP, form an enclosed catalytic chamber to entrap and degrade monomeric amyloidogenic peptides to prevent the formation of toxic aggregates ${ }^{6}$. However, the structure of the catalytic chamber in the PreP closed state precludes the capture of its substrate such as $A \beta$, or the release of its reaction products, key steps in the PreP catalytic cycle (Extended data Fig. 1). To date, no structure of an open state of M16C clan of metalloprotease has been reported. Solution scattering studies indicate that human PreP in solution is mostly in a closed or partially closed state ${ }^{6}$. However, the structural basis for the closed-open transition that allows for substrate capture and release of proteolysis products, in addition to substrate-induced transition from open to closed state remains elusive (Extended data Fig. 1). Here, we integrate cryoEM, crystallography, size-exclusion chromatography (SEC) coupled small angle X-ray scattering (SAXS), hydrogen deuterium exchange (HDX)-mass spectrometry (MS), site-specific mutagenesis, and chemical biology approaches to elucidate the structural basis of the open-closed transition and the mechanism of substrate recognition for human PreP.

\section{Results}




\section{Solution of multiple open and substrate-bound closed structures by cryo-EM}

Advances in cryo electron microscopy (cryoEM) allow the structural determination of conformational states recalcitrant to the crystallography ${ }^{23-26}$, thus we used cryoEM to examine the conformational states of PreP in the absence of substrate (apo-PreP). We first used differential scanning fluorimetry (DSF) to optimize the unfolding entropy and unfolding and dissociation enthalpy of human PreP (Extended data Fig. 2) ${ }^{27}$. Apo-PreP grids were then prepared by Vitrobot and a dataset of 2,626 micrographs collected at a $300 \mathrm{kV}$ on a Titan Krios at various ice thicknesses to overcome orientation bias and/or denaturation by exposure to the air-water interface (Table 1, Extended Fig. 3,4). Apo-PreP grids were then prepared by Vitrobot and a dataset of 2,626 micrographs collected at $300 \mathrm{kV}$ on a Titan Krios. During processing, we observed that the predominant classes contained particles only half the expected size of PreP (Fig. 1A, Extended data Fig. 3). Following 3D classification of 411,000 particles, four classes were obtained (Extended data Fig. 3). The two major classes, comprising 208,000 and 118,000 particles, displayed an intact PreP-N domain but a denatured PreP-C domain and were refined to $4.2 \AA$ (Fig. 1B, Extended data Fig. 4,5). The third class, comprised of 50,000 particles and refined to $4.5 \AA$, was found to contain full-length PreP adopting a partial open conformation that we designate $\mathrm{pO}$ (Fig. 1B, Extended data Fig. 3). The final class, of $~ 34,000$ particles, was comprised of nearly full-length PreP particles adopting an open conformation was refined to $5.3 \AA$ (Extended data Fig. 3).

It has previously been demonstrated that the vast majority of particles derived from diverse proteins or protein complexes (90\%) are adsorbed to the air-water interface ${ }^{28}$. Extensive studies have shown that many proteins are denatured rapidly upon exposure to the air-water interface ${ }^{29}$. Therefore, we hypothesized that the extensive denaturation of the PreP-C domain described above resulted from denaturation at the air-water interface. To explore this possibility, we used fiducial-less cryo electron tomography (cryoET) to examine the distribution of PreP particles within the vitrified ice. This analysis of the Vitrobot prepared grids revealed that nearly all PreP particles were adsorbed to the air-water interface (Fig. 1C, Extended data Fig. 6, Movie 1). Approximately 88\% of particles had half of the anticipated size in our cryoET analysis, consistent with that a majority of $3 \mathrm{D}$ classes $(80 \%)$ having a denatured PreP-C domain (Fig. 1C, Extended data Fig. 6A). Interestingly, while PreP-N and PreP-C share a highly similar structure, PreP-N has an additional $\beta$-hairpin (Extended data Fig. 6B). This $\beta$-hairpin extends from an $\alpha-$ helix that binds the catalytic zinc ion and interacts with the a-helical hairpin that links PreP-N and PreP-C. This unique structure likely makes PreP-N more stable than PreP-C (Extended data Fig. 6B). Together, our data indicate that PreP-C preferentially denatured during the vitrification process, either by the repetitive exposure to air-water interface and/or shear force caused by filter blotting (Extended data Fig. $6 \mathrm{C})^{30}$.

We hypothesized that if the amount of time the sample spent on the grid prior to vitrification (dwell time) could be significantly reduced, PreP denaturation would likewise be reduced. Spotiton, a novel method of vitrifying samples using a piezo electric dispensing head to deliver sample droplets onto a self-blotting nanowire grid, has been shown to significantly reduce the dwell time of particles at the air-water interface 
prior to vitrification ${ }^{30-33}$. We employed this technique to prepare grids using a 133 millisecond dwell time (compared to 1-2 seconds for Vitrobot) by chameleon ${ }^{34}$, a commercial version of Spotiton developed by SPT Labtech via the National Center for CryoEM Access and Training (NCCAT) general user program 2. An apo-PreP dataset of 3,012 micrographs was processed to yield about 363,000 particles from welldefined 3D classes from these grids. Following 2D and 3D classification, all classes were found to contain full-length PreP particles, and no denaturation was observed (Fig. 1D, 1E, Table 1, Extended data Fig. 7,8). 3D classification of PreP particles revealed three distinct open state structures of PreP. They were refined to an open state ( 0 ) and two partially open states (p01 and p02) with resolution of $4 \AA$, $3.7 \AA, 3.9 \AA$, respectively. Structural models of these three states were then built and refined (Table 1 , Extended data Fig. 5B-D, 8, Movie 2). Two substrate-bound PreP cryoEM structures were also determined. We also optimized PreP in the presence of a five-fold molar excess of A $\beta$ 1-40 by DSF. Grids were prepared by chameleon, and a dataset of 3,483 micrographs was processed to yield about 175,000 particles from a well-defined 3D class that was refined to a partially closed $(\mathrm{pC})$ state of PreP at $3.3 \AA$ resolution (Fig. 1F, Table 1; Extended data Fig. 5E,9,11). A similar approach was used to obtain a Coulomb potential map for PreP in complex with a model presequence peptide from human citrate synthase (27 aa long, CS27) ${ }^{35}$, resulting in a pC state PreP at $4.6 \AA$ resolution (Fig. 1G, Table 1; Extended data Fig. 10,11). Structural models of these substrate-bound PreP were then built and refined (Table 1, Extended data Fig. 5E,11, Movie 3). We define the substrate-bound PreP cryoEM structures as pC state to differentiate from the closed (C) state crystal structures of PreP that were solved in the presence or absence of $A b^{6}$.

\section{Structural analysis of apo- and substrate-bound PreP reveal key states in PreP catalytic cycle and the molecular basis for substrate recognition}

Comparison of the five cryoEM structures derived from the apo- and substrate-bound PreP reveals three key conformational states based on the degree of opening of the catalytic chamber: open ( 0 ), partially open ( $\mathrm{pO}$ ), and partially closed ( $\mathrm{pC}$ ). Apo-PreP has three states, $\mathrm{O}, \mathrm{p01}$, and $\mathrm{pO} 2$ that are distinct from each other with the two $\mathrm{pO}$ states being slightly more open than the $\mathrm{pC}$ or $\mathrm{C}$ states (Fig. 2A, Extended data Fig. 12, Table S1). A $\beta$ - and citrate synthase presequence (CS27)-bound PreP structures in the pC state are nearly identical to each other and are slightly more open than crystal structures of apo or Ab-bound PreP6 (Fig. 2A, Extended data Fig. 12, Table S1). Of these structures, the pO, pC, and C states of PreP have a small variation in the degree of opening, making their chamber inaccessible to substrate binding. Thus, only PreP open state has a large enough opening to capture its peptide substrates and release the proteolytic products.

Two major differences exist between these structures. The first is mediated by the rigid body displacement between PreP-N and PreP-C domains, whereby the two halves of this chamber-containing protease open up, similar to a clamshell (Fig. 2A-C, Extended data Fig. 12, Movie 4, Table 1). The 
displacement results in a difference in the distance, angle, and contacts between these domains. Most noticeably, both the distance and angle between PreP-N and PreP-C in PreP open state is substantially larger than the rest of states while the buried surface between PreP-N and PreP-C is much reduced compared to the others. The displacement between PreP-N and PreP-C is most likely driven by the entropically favorable rigid body motion. We also observed major conformational rearrangements in two additional regions, switch A (aa 174-225) and switch C (aa 506-550) (Fig. 2A-C; Extended data Fig. 12, Movie 4). Switch A contains two a-helices that have residues for the binding of substrate and catalytic zinc ion (Fig. 2A). The helix-turn-helix motif of the switch $\mathrm{C}$ region joins PreP-N and PreP-C and makes extensive contacts with an extended $\beta$-hairpin within the long a-helix of the switch $A$ region, allowing the switch $A$ and $C$ region move jointly with the displacement between PreP-N and PreP-C (Fig. 2A).

3D classification revealed that the majority of PreP particles $(\sim 74)$ were in the partially open p0 states, indicating that PreP in the absence of substrate prefers to be in a state inaccessible to substrate binding. We then used size exclusion chromatography (SEC) in-line with small angle X-ray scattering (SAXS) to further assess how the distribution of PreP conformational states in solution is influenced by the presence of $A \beta$ and presequence from citrate synthase (CS27) under physiological buffer conditions (Fig. 2D, Extended data Fig. 13, Table 2). SAXS is a highly effective technique to eliminate structural models that do not produce calculated scattering patterns that fit the experimental scattering profile ${ }^{36}$. SEC-SAXS also reduces large aggregates that contaminated our previously reported SAXS profile of PreP6 ${ }^{6}$. Consistent with the cryoEM data, the SEC-SAXS data confirms that PreP alone in solution is also preferred in a pO state $(\sim 63 \%)$, instead of the open state $(\sim 37 \%)$ (Extended data Table 2$)$. The fact that PreP in solution prefers the pO state rather than the open state is logical because the transition from the pO states to 0 state loses substantial buried surface (650-940 $\AA^{2}$ ) and a network of hydrogen bonds and salt bridges, and thus is energetically unfavorable (Extended data Table S1). The presence of CS27 significantly reduced the $\mathrm{R}_{\mathrm{g}}$ value from $31.5 \AA$ to $30.8 \AA$, leading PreP to be mostly in the closed state ( $82 \%$ ) (Extended data Table 2). However, the presence of $A \beta$ only slightly reduced the $R_{g}$ and $D_{\max }$ values. This is consistent with that only a subtle conformational switch occurs between $\mathrm{pO}$ and $\mathrm{pC}$ states (Fig. 2D, Extended data Fig. 13, Table 1), whereby substrate-binding promotes domain closure.

Together, our data leads to the hypothesis that PreP undergoes the following conformational switch during the catalytic cycle (Fig. 2E): PreP is predominantly in the partial open state at the resting condition. The transition from the $\mathrm{pO}$ states to $\mathrm{O}$ state allows the capture of substrate, and thus is a key state in the catalytic cycle. Upon opening, peptides that are rich in positively charged residues are attracted to the negatively charged, catalytic chamber of PreP-N which can further select for its substrates based on their size and conformational compatibility within the chamber (Fig. 2F) ${ }^{6}$. The catalytic site of PreP undergoes minimal conformational change, and thus is poised to carry out proteolysis (Fig. 2G). After proteolysis, the closed to open transition allows the release of proteolytic products to initiate the next catalytic cycle. 


\section{Mechanism for the conformational switches between PreP open and closed states in the presence and absence of substrates}

The comparison of PreP cryoEM structures reveals the molecular basis for the equilibrium between partially open and open state in the absence of substrate. PreP has three regions that undergo substantial conformational switches, defined as switch A, B, and C (Fig. 3A). As discussed above, switch $A$ and $\mathrm{C}$ move together with rigid body displacement between PreP-N and PreP-C (Fig. 2A, 3A). The switch $A$ and $C$ regions in the $p O$ states have lower resolution and higher thermal $B$ factors than the rest of PreP structures (Fig. 3B). In comparison, the resolution and thermal B factors of these regions in the open and substrate-bound states are not profoundly different from the rest of protein (Fig. 3B). Together, this is indicative of high conformational dynamics within these regions. As switch A contains residues involved in substrate peptide binding and catalytic zinc ion coordination, such high dynamic would render pO state catalytically incompetent. The presence of substrate stabilizes switch $A$, and thus, the residues for substrate binding and catalysis, allowing the catalytic reaction. This suggests that PreP uses the substrate-assisted catalysis as a mechanism for the substrate selection. This is because amyloid peptides have the high propensity to unfold and form a b-strand, which can then form the cross-b-sheet with other amyloid peptides. The catalytic cleft of PreP selectively binds the b-strand of substrate peptide after such peptide is unfolded inside the catalytic chamber. Only peptides that tend to unfold and form the b-strand can stabilize PreP's catalytic cleft, leading to catalysis.

PreP switch B (aa 421-436, within PreP-N) represents a rotation of an a-helix when the PreP open state is compared with the rest of conformational states (pO, pC, or C) (Fig. 3C,3D; Extended data Movie 5). The rotation of switch $B$ is particularly noticeable at residue $M 436$, which rotates $\sim 60^{\circ}$ to transit through the hydrophobic pocket formed by $\mathrm{Y} 450$ and L467 (Fig. 3C,3D). This allows a rigid body rotation of PreP-C in relationship to PreP-N (Fig. 2E, 3E). Such a rotation maintains the interactions between switch $B$ and PreP-C, e.g., the contacts of E432 with R675 and that of H430 with N676 and the main chains of aa residues 642 and 643. Thus, there should be minimal energetic barrier for such rotation to allow the rapid shift between PreP partially open and open states driven by the entropically favorable rigid body motion between PreP-N and PreP-C.

The rotation of switch B also offers the molecular basis for PreP's substrate-induced conformational switch and substrate selectivity. Switch B has hydrophobic residues L428 and 1432 that, together with the

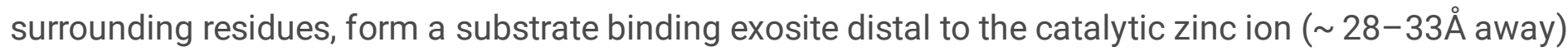
(Fig. 3F, Extended data Movie 5). This exosite is highly hydrophobic and is shown to bind $A \beta$ residues that are away from the preferred cleavage sites of $A \beta$ (aa $19 / 20$ or aa 20/21) (Fig. 3F) ${ }^{6}$. The interaction of the exosite with the hydrophobic residues of substrate would favor the closed state and thus promote the open to closed transition (Fig. 3E). Furthermore, this in part explains why PreP prefers to degrade peptide substrates that are rich in hydrophobic residues, e.g., presequences and $A \beta$. 
Our structural analysis predicts the conformational dynamics of switch $\mathrm{A}$ and $\mathrm{C}$ and substrate-induced stabilization of three switch regions. To test this, we utilized peptide amide hydrogen deuterium exchange (HDX) mass spectrometry, which is a powerful tool to probe protein conformational dynamics because it allows evaluation of comparative solvent accessibility throughout the protein ${ }^{36-41}$. As expected, a reduced HDX was observed in segments in both PreP-N and PreP-C that are involved in the substrate binding (e.g., aa 91-141, aa 703-720, aa 892-903, and aa 931-940). We also observed reduced HDX of both switch $A$ and $C$ regions and, to a lesser degree, switch $B$ when two different PreP substrates, $A \beta$ and citrate synthase presequence (CS27) were present, which confirmed our prediction (Fig. 3G, Extended data Fig. 14-16). To probe how the interface between PreP-N and PreP-C controls the equilibrium between the $\mathrm{pO}$ and $\mathrm{O}$ states, we carried out structure-guided mutagenesis in this region at positions predicted to weaken the interaction between PreP-N and PreP-C. We found that two point mutations, D367A and Q637A modestly increased the catalytic activity of PreP (Fig. 3H). Thus, destabilization of this interface enhances, rather than diminishes, PreP's enzymatic activity, presumably through increasing the ease with which PreP can transition through the key conformational states of its catalytic cycle.

\section{Mechanism of inhibition of MitoBlocker-60 (MB-60)}

At the PreP-N and -C interface, we observed an intriguing overlap between a key conformational switch (B) and a key substrate-binding site, the exosite, whose functional role in substrate-binding and catalysis is largely unexplored. To further define the conformational dynamics at this interface, we exploited the findings of an in vitro high-throughput screen that identified MB-60 (1-(diphenylmethyl)-4-(3-methyl-4nitrobenzoyl)piperazine) as a potent inhibitor of PreP (Fig. 4A). MB-60 inhibited the degradation of $A \beta$ by PreP with an $\mathrm{IC}_{50}=200 \mathrm{nM}$ and triggered mitophagy under mitochondrial stress ${ }^{42}$. A previous CRISPRi screen showed that PreP was essential for the robust cell proliferation of human K562 leukemia cells ${ }^{43}$. Consistent with this notion, MB-60 potently blocked cell proliferation in a dose dependent manner without inducing cell death (Fig. 4A). To understand the mechanism of inhibition, we co-crystallized MB-60 with human PreP. The structure of MB-60-bound PreP at $2.3 \AA$ resolution reveals an unexpected binding mechanism of MB-60 (Fig. 4B, Table 2, Extended data Fig. 17). In the presence of MB-60, PreP exists in a closed conformation that is nearly identical to structures of substrate-free and $A \beta$-bound PreP (RMSD = $0.15 \AA$ and $0.31 \AA$, respectively $)^{6}$. Within the catalytic chamber, two MB-60 molecules wrap around each other to make intimate interactions to bury $313 \AA^{2}$ and bind the distinct pockets at the PreP exosite via various contacts to bury $808 \AA^{2}$ and $658 \AA^{2}$ surfaces of MB-60-a (pink) and MB-60-b (yellow), respectively (Fig. 4B,4C). For MB-60-a, two phenyl groups bind a hydrophobic pocket in close contacts with L60, F344, 1432, M446, L447, and L467. The carbonyl group of MB-60-a forms hydrogen bonds with waters coordinated by the carbonyl group of M446 and hydroxyl group of Y383. The piperazine group of MB-60a forms hydrogen bond with water coordinated with the side chain of Q435. The nitro group of MB-60-a forms a hydrogen bond with the main chain of G382 and a cation- $\pi$ interaction with Y383. For MB-60-b, two phenyl groups interact with the hydrophobic pocket formed by I337, A343, F344, 1451, and L464 while 
the nitro group of MB-60-b forms a salt bridge with K431. The finding that MB-60 targets PreP's exosite provides strong evidence that this site plays a critical role in PreP catalysis.

To explore how MB-60 affects the substrate-binding and conformational dynamics of PreP, we first used SEC-SAXS and showed that MB-60 did not induce obvious change in the SAXS profile (Fig. 4E, Extended data Fig. 13). The effect of MB-60 on the conformational dynamics of PreP was then explored by the differences between amide H/D exchange profiles of PreP alone and PreP in the presence of MB-60. Most exchanges were unchanged (Fig. 4F, Extended data Fig. 14-15). Of a few regions that showed noticeable reduction in H/D exchange, the peptides around MB-60 binding pockets were most prevalent. These included residues 349-364, 371-388, and 415-478 (Fig. 4F, Extended data Fig. 18). This supports the notion that MB-60 in solution binds the hydrophobic pockets revealed by our MB-60-bound PreP structure. While the reduced exchanges in the switch $\mathrm{B}$ region is expected as it is a part of MB-60 binding sites, segments in switch $A$ and switch $C$ regions (aa 201-213 and aa 503-522, respectively) that are away from MB-60 binding site also displayed reduced H/D exchange (Fig. 4F, Extended data Fig. 18). This is consistent with our model that in the absence of MB-60, the switch $A$ and $C$ regions of PreP undergo dynamic motion. Moreover, that binding of MB-60 to the exosite stabilizes such motion suggests that conformational changes in the exosite are functionally coupled to those in switch $A$ and $C$. It is worth noting that, similar to the binding of substrates, $A \beta$ and CS27, segments in PreP-C that are near the catalytic site also had the reduced exchanges (e.g., aa 703-720, aa 892-903, and aa 931-940, Fig. 4F). This is consistent with our model that the binding of MB-60 to the switch $B$ region can further stabilize pO states, leading to a better interaction between PreP-N and PreP-C.

Together, our structural and HDX-MS analysis explains how MB-60 prevents degradation of $A \beta$ and other substrates. MB-60 is too large in size to enter the catalytic chamber of PreP when PreP is in pO state, the dominant state in solution. Upon entering into the catalytic chamber via the PreP open state, MB-60 should promote the rotation of switch B, promoting the open to closed transition. Such interaction should disfavor the $\mathrm{pO}$ to $O$ transition, preventing the access of $A b$ to the catalytic chamber. Furthermore, MB- 60 also blocks $A \beta$ from binding to PreP exosite even after $A b$ enters into PreP catalytic chamber. The close interactions of PreP with both phenyl groups of MB-60 also explains the structure-activity relationship of MB-6042: loss of one phenyl group increased the $\mathrm{IC}_{50}$ value five-fold while the relocation of the nitro group within the tolyl group of MB-60 increased the $\mathrm{IC}_{50}$ value 25 -fold.

\section{Discussion}

Major advances in instruments, techniques, and methods have fueled a "resolution revolution", making single particle cryoEM a powerful structural determination technique that rivals macromolecular

crystallography ${ }^{23-26}$. However, rapid protein denaturation in the thin film generated during the grid making process, due to the high surface area to volume ratio at the air-water interface, represents a major obstacle in identifying the suitable condition to vitrify protein sample for cryoEM analysis ${ }^{29}$. Our cryoET analysis reveals that PreP, a $117 \mathrm{kDa}$, monomeric enzyme with homologous $55 \mathrm{kDa} \mathrm{N}$ - and C-domains 
preferentially absorbed to the air-water interface. Such exposure likely caused preferential denaturation of the PreP-C domain. Attempts in identifying better buffers using DSF, adding substrate, e.g., Ab to lock PreP in the closed state, or altering the grid properties, e.g., grid with hydrophilized graphene, all failed to properly vitrify PreP (data not shown) ${ }^{27,44}$. The ability of the PreP-N domain to withstand exposure to the air-water interface, despite its high degree of structural homology to the PreP-C domain, is likely due to enhanced stability granted by the lock formed by switch A and switch $\mathrm{C}$ regions (Extended data Fig. 6). The combination of self-blotting nanowire grids and piezo dispensing utilized by chameleon, the commercial version of Spotiton, eliminated the paper blotting step and significantly reduced the time for PreP to interact with the air-water interface ${ }^{30-34}$. This led to the successful determination of apo- and substrate-bound PreP cryoEM structures. Thus, PreP is a compelling case study that demonstrates how reduced vitrification times afforded can be used to alter the kinetics of protein denaturation at the airwater interface that has led to the near atomic resolution $(3.3 \AA-4.6 \AA)$ cryoEM structures of a relatively small protein (117 kDa human PreP) that exhibits high conformational heterogeneity. Therefore, PreP represents an ideal model protein to assess the efficacy of newly emerging theories and practices of grid chemistry and vitrification process, e.g., VitriJet or shake-it-off, aimed at preventing protein denaturation during vitrification ${ }^{45,46}$.

Our integrative structural approaches lead to the formulation of the following model for the catalytic cycle of PreP (Fig. 5A). Both cryoEM and SAXS data indicate that apo-PreP prefers to be in the partially open state, which cannot capture substrates. The C-terminal end of the switch B helix (e.g., Met 436) is located at the region where PreP-C pivots away from PreP-N during the closed to open transition (Fig. 3D, 5A). The rigid body motion of PreP-N and PreP-C entropically drives the separation between PreP-N and PreP-C domains. Governed by the rotation of the switch B region, the rigid body motion between PreP-N and PreP-C can trigger the conformational switch of the extended loop in the switch $\mathrm{C}$ region, allowing PreP to transition into the open state (Fig. 3C-E, 5A). Presequences, rich in positively charged residues can then be attracted to the negatively charged surface of the PreP-N catalytic chamber (Fig. 2F, 5A).

Furthermore, the high dipole moment of $A \beta$ permits the charge complementation of this peptide with the catalytic chamber formed by PreP-N and PreP-C, which are negatively and positively charged, respectively (Fig. 2F, 5A). Plentiful hydrophobic residues in presequences and $A \beta$ or the small molecule inhibitor, MB60 then interacts with the hydrophobic exosite to promote the rotation of switch $B$ helix that induces the open to closed transition (Fig. 3F, 5A). The hydrophobic residues of PreP substrates also interact with the hydrophobic pocket at the catalytic site formed by PreP-N and PreP-C, further promoting the favorable interaction between PreP-N and PreP-C (Fig. 3F, 5A). The motion between PreP open and closed states, in conjunction with the selective interaction between the PreP catalytic chamber and the peptide substrate, provide the requisite force to unfold presequences and $A \beta$. This leads to the exposure of a $\beta$-strand within the presequence peptide or $A \beta$ that can complement and stabilize the catalytic cleft formed in part by the switch A region, which in turn facilitates proteolysis. PreP then transitions from the closed state to the open state to release the reaction products. The transition between PreP open and partially open/closed state occurs quite frequently because its rate needs to be faster than the rate of $A \beta$ degradation, which is 50-200 per second ${ }^{6}$. The model described above provides the molecular basis for the key

Page $11 / 30$ 
conformational changes during the PreP catalytic cycle that facilitate amyloidogenic peptide capture and degradation. As the loss of function mutations in human PreP are associated with neurological disorders, e.g., cognitive impairments/disability and cerebellar atrophy ${ }^{10,11}$, our model should provide guidance for future investigation into how to boost PreP activity for better control of mitochondrial proteostasis.

Our structural analysis of two members of the cryptidase family, IDE and PreP suggests a common framework for amyloidogenic peptide recognition with distinct specializations that support efficient cytotoxic peptide clearance in their distinct cellular niches ${ }^{6,22,36,47,48}$. Both enzymes belong to the M16 clan of metalloproteases and have homologous $\sim 55 \mathrm{kDa} \mathrm{N}$ - and C-terminal domains (Fig. 5) ${ }^{22,48}$. Both enzymes also undergo the open-closed transition during their catalytic cycle and only the open state can

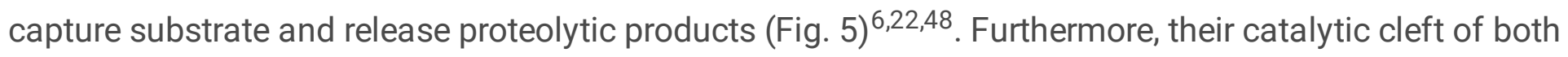
enzymes is formed between their $\mathrm{N}$ - and $\mathrm{C}$-terminal domains, which is unstable in the absence of substrate. They use substrate-assisted catalysis mechanism to selectively recognize and degrade amyloid peptides (this work) ${ }^{36,47}$. However, there are noticeable differences between IDE and PreP. Firstly, how these two enzymes open up is quite different due to the connecting region between their $\mathrm{N}$ - and $\mathrm{C}$ terminal domains (Fig. 5). PreP is connected by the relatively long, dual a-helical hairpin switch $\mathrm{C}$ region that is connected to the zinc-containing D1 domain via the switch A region. Consequently, PreP undergoes a "book-opening" motion along a rather large surface between the PreP-N and PreP-C domains that is guided by the rotation of the switch $B$ region. However, the short $\sim 40$ amino acid long loop of IDE allows IDE-N and IDE-C domains to pivot along a much smaller surface between D2 and D3 domains for a "packman-like" open-close motion, leading to the maximal separation between D1 and D4 domains 22 . The second difference is their oligomerization state (Fig. 5). The monomeric PreP 'rests' in a partially open state and the transition between partial open and open state allows PreP to capture its substrates. Contrary to monomeric PreP, IDE exists as a dimer to allow for the allosteric regulation of catalysis ${ }^{36,47}$. Specifically, at least one of the two subunits within the IDE dimer is typically in the open state and primed for substrate capture most of the time. The binding of substrate allosterically facilitates the opening of the other IDE subunit within the IDE dimer, leading to enhanced IDE catalysis ${ }^{36,47}$. Furthermore, these two enzymes use the exosite located at their D2 domain to recognize different structural features of their substrates (Fig. 5). The PreP exosite recognizes hydrophobic residues of peptide substrates while the IDE exosite binds the $\mathrm{N}$-terminal mainchains ${ }^{6,49}$. Despite these profound differences, we have shown that the structural analyses can be used to rationally design mutations to enhance the enzymatic activities of both enzymes (this work) ${ }^{47}$. Furthermore, small molecules that can either boost IDE activity or selectively inhibit IDE to degrade insulin has been discovered ${ }^{50-52}$. Future work will realize the preventative or therapeutic potential in controlling the proteolytic activity of $A \beta$-degrading proteases for neurodegenerative diseases caused by amyloid peptide-mediated toxicity.

\section{Materials And Methods}

\section{Expression and Purification of PreP}


Wild type human PreP and various mutants were expressed and purified as described ${ }^{6}$. Briefly, E. coli Rosetta(DE3) containing the expression plasmid for the desired PreP construct was grown at $25^{\circ} \mathrm{C}$ with $300 \mu \mathrm{M}$ IPTG induction for $20 \mathrm{hrs}$. Proteins were then purified over Ni ${ }^{2+}-\mathrm{NTA}$ affinity, Source $\mathrm{Q}$ anion exchange, and Superdex 200 size exclusion columns, flash-frozen in liquid nitrogen and stored at $-80^{\circ} \mathrm{C}$.

\section{Differential scanning fluorimetry}

To optimize conditions for cryo-EM data collection, differential scanning fluorimetry was applied to screen 40 buffers and 98 additive conditions. The DSF was carried on with about $1 \mathrm{mg} / \mathrm{ml}$ PreP and 10X Sypro Orange in $20 \mu \mathrm{l}$ buffers using Thermo Fisher Step ONE RT-PCR. Using the melting temperature and slope as selection criteria, the following condition was identified as best for the grid making with $20 \mathrm{mM}$ Tris (pH 7.7), $150 \mathrm{mM} \mathrm{NaCl}, 10 \mathrm{mM}$ EDTA, $0.5 \mathrm{mM} \beta$-mercapto-ethanol.

\section{CryoEM data collection and analysis}

Purified PreP was further purified by Superdex 200 chromatography using buffer containing $20 \mathrm{mM}$ Tris, $\mathrm{pH}$ 7.7, $150 \mathrm{mM} \mathrm{NaCl}, 10 \mathrm{mM} \mathrm{KCl}, 20 \mathrm{mM}$ EDTA and $1 \mathrm{mM} \beta$-mercaptoethanol. Grids were prepared using either Vitrobot or chameleon ${ }^{34}$. For Vitrobot, Quantifoil holey carbon coated 200 mesh copper R1.2/1.3 grids were plasma cleaned using air for 30 secs using a Solarus plasma cleaner (Gatan). $2.5 \mu$ l of sample was applied to the grid, then 15 secs allowed to pass elapsed before blotting with one layer of filter paper, force $1,100 \%$ humidity, for $2.5-3.5$ secs from both sides of the grids followed immediately by plunging into liquid ethane. For chameleon prepared grids, 300 mesh carbon or gold lacey nanowire grids were plasma cleaned with $\mathrm{O} 2$ and $\mathrm{H} 2$ for 10 secs using a Solarus plasma cleaner (Gatan). The grids were plunged at 133 milli-second. All images were acquired using a Titan Krios microscope (FEI) operated at $300 \mathrm{KeV}$ with a Gatan K2 direct electron detector (Gatan) in counting mode. Images were automatically acquired using Leginon ${ }^{53}$ using collection parameters as shown in Table 1. Images were processed using software integrated into the RELION3.0 ${ }^{54}$. Frames were aligned using MotionCor2 software with dose weighting ${ }^{55}$, CTF was estimated using Gtf $^{56}$, particles were picked and extracted automatically using RELION3.0 ${ }^{54}$. Particle stacks were processed through several rounds of 2D and 3D classification.

Example images and 2D class averages are shown in Figure S3, S7, S9, and S10. Selected classes were then processed for high-resolution 3D refinement (Figure S3, S7, S9, S10). Finally, the overall map was improved by particle polishing in RELION3.054. The final resolution was estimated using gold-standard Fourier Shell Correlation (FSC $=0.143$ ) (Figure S4, S8, S9, S10). CryoEM data collection and processing statistics are listed in Table 1. Structural models were built using high resolution PreP structure (PDB = $4 N G E)$ as a template. Density fitting and structure refinement was performed using UCSF CHIMERA ${ }^{57}$, $\mathrm{COOT}^{58}$, and PHENIX ${ }^{59}$. The refinement statistics are listed in Table 1.

\section{CryoET analysis}


Tilt-series were collected with Legion ${ }^{53}$ on a Titan Krios with a Gatan K2 counting camera using the same sample preparation as for cryoEM. Tilt-series were aligned with Appion-Protomo ${ }^{60}$ and reconstructed with Tomo3 $\mathrm{D}^{61}$. Sub-tomogram processing-including particle picking, alignment, and classification-was performed with Dynamo ${ }^{62}$.

\section{Protein crystallization, data collection, and structure determination:}

PreP E107Q was modified by reductive lysine methylation prior to Superdex 200 chromatography. 5$7 \mathrm{mg} / \mathrm{ml}$ lysine-methylated PreP-E107Q in buffer containing $20 \mathrm{mM} \mathrm{HEPES} \mathrm{pH} \mathrm{7.5,} 250 \mathrm{mM} \mathrm{NaCl}, 2 \mathrm{mM}$ DTT, and $200 \mu \mathrm{M}$ MB-60 was combined with mother liquor containing 15.0\% (w/v) PEG 8,000, $15 \mathrm{mM}$ TCEP, $80 \mathrm{mM}$ sodium cacodylate $\mathrm{pH} 6.7,160 \mathrm{mM}$ calcium acetate, and $20 \%(\mathrm{v} / \mathrm{v})$ glycerol in a 1:1 (v/v) ratio for the crystallization of PreP-MB-60 complex by hanging-drop vapor diffusion at $18{ }^{\circ} \mathrm{C}$. Crystals grew for one week prior to data collection. Crystals were cryoprotected in mother liquor containing $30 \%$ $(\mathrm{v} / \mathrm{v})$ glycerol, then flash frozen in liquid nitrogen. Diffraction data were collected at beamline 19ID at Argonne National Laboratory and processed using HKL300063. The structure of PreP in complex with MB60 was determined by molecular replacement using Phaser and PreP structure (4L3T) as the search model. Model building - including the addition of missing 317-323 residues in chain A-were performed using $\mathrm{COOT}^{58}$ and refinement was done using PHENIX ${ }^{59}$. The final $2.27 \AA$ resolution model $(\mathrm{pdb}=4 \mathrm{RPU})$ has $R_{\text {work }}=18.6 \%$ and $R_{\text {free }}=20.6 \%$. Data collection and structure refinement statistics are listed in Table 2 . Presumably due to the shorter time of crystallization, only cysteine 112 was modified with the dimethylarsenic moiety while cysteine 556 was not. Cysteine 556 is in the close proximity with cysteine 119 to form a disulfide bond. The absence of disulfide bond between these residues might be due to the presence of reducing agent during the purification and/or crystallization.

\section{SAXS Data Collection and Analysis}

SAXS data were collected at Argonne National Laboratory's Advanced Photon Source, beamline 12ID-B, at $23^{\circ} \mathrm{C}$ using $1.1 \mathrm{mg} / \mathrm{ml}$ protein and an incident X-ray wavelength of $0.886 \AA$, and protein concentration of $0.5 \mathrm{mg} / \mathrm{ml}$. For MB-60-binding experiments, PreP was preincubated with $200 \mu \mathrm{M}$ MB-60 on ice prior to SAXS data collection. Data was reduced and analyzed using ATSAS ${ }^{64}$. PRIMUS and GNOM in the ATSAS suite were used to determine the $R_{g}$ value in reciprocal and real space, respectively ${ }^{64}$. $D_{\max }$ and $P(r)$ distribution were calculated by GNOM. Theoretical scattering curves for different models were generated and fit to the experimental data using CRYSOL in ATSAS suite ${ }^{64}$. OLIGOMER in ATSAS suite was used to determine the percent composition by parsimonious conformational states that best fit the observed data ${ }^{64}$. 


\section{Deuterium exchange mass spectrometry (DXMS)}

Prior to performing comparative $\mathrm{H} / \mathrm{D}$ exchange experiments, enzymatic and quench conditions that produced an optimal fragmentation pattern of PreP were established as previously described ${ }^{65}$. Briefly, for PreP and MB-60 studies, $3 \mu \mathrm{l} 4.7 \mathrm{mg} / \mathrm{ml}$ PreP in buffer containing $20 \mathrm{mM}$ Tris- $\mathrm{HCl}(\mathrm{pH} 7.5)$, and $50 \mathrm{mM} \mathrm{NaCl}$ was diluted with $9 \mu$ l of buffer $\mathrm{A}\left(8.3 \mathrm{mM}\right.$ Tris- $\mathrm{HCl}(\mathrm{pH} 7.5), 50 \mathrm{mM} \mathrm{NaCl}$ in $\mathrm{H}_{2} \mathrm{O}$ ) at $0^{\circ} \mathrm{C}$. For PreP and substrates study, $3 \mu 10.5 \mathrm{mg} / \mathrm{ml}$ PreP in buffer containing $20 \mathrm{mM}$ Tris- $\mathrm{HCl}(\mathrm{pH} 7.7), 150 \mathrm{mM}$ $\mathrm{NaCl}$ and $10 \mathrm{mM}$ EDTA was diluted with $9 \mu \mathrm{l}$ of buffer $\mathrm{B}(8.3 \mathrm{mM}$ Tris- $\mathrm{HCl}(\mathrm{pH} 7.7), 150 \mathrm{mM} \mathrm{NaCl}, 10 \mathrm{mM}$ EDTA in $\mathrm{H}_{2} \mathrm{O}$ ) at $0^{\circ} \mathrm{C}$. The sample was then mixed with $18 \mu \mathrm{l}$ of ice-cold quench buffers containing $0.8 \%$ formic acid, $16.6 \%$ glycerol and various concentrations of $\mathrm{GuHCl}(0.08,0.8$ and $1.6 \mathrm{M})$. Quenched samples were then subjected to DXMS for proteolysis and LC/MS analysis. The use of $0.8 \mathrm{M} \mathrm{GuHCl}$ resulted in the best sequence coverage of PreP. For DXMS analysis, $13 \mu \mathrm{M}$ PreP in the presence or absence of $130 \mu \mathrm{M}$ MB-60 with buffer $8.3 \mathrm{mM}$ Tris-HCl pH7.2, $50 \mathrm{mM} \mathrm{NaCl}$, and 2.1\%DMSO in $\mathrm{H}_{2} \mathrm{O}$, $260 \mu \mathrm{M} \mathrm{A} \beta$, or $260 \mu \mathrm{M} \mathrm{CS} 27$ in buffer $\mathrm{B}$ and $2.1 \% \mathrm{DMSO}$ in $\mathrm{H}_{2} \mathrm{O}$ was incubated at room temperature for $30 \mathrm{~min}$ prior to chilling to $0^{\circ} \mathrm{C}$ for deuteration studies. Functional hydrogen-deuterium exchange reactions were initiated by adding $3 \mu \mathrm{l}$ sample into $9 \mu \mathrm{l}$ of buffer $A$ or buffer $B$ in $D_{2} O\left(p D_{R E A D}=7.2\right)$ and incubated at $0^{\circ} \mathrm{C}$ for $10,100,1,000,10,000$, and $100,000 \mathrm{sec}$ and at room temperature for $100,000 \mathrm{sec}$ (representing $1,000,000 \mathrm{sec}$ at $0^{\circ} \mathrm{C}$ based on 10 -fold enhanced exchange rate at room temperature) ${ }^{66}$. The exchange reaction was terminated by adding $18 \mu \mathrm{l}$ of ice-cold $0.8 \%$ formic acid, $0.8 \mathrm{M} \mathrm{GuHCl}, 16.6 \%$ glycerol for a final $\mathrm{pH}$ of 2.5. Quenched samples were then immediately frozen on dry ice and stored at $-80^{\circ} \mathrm{C}$ prior to LC/MS analysis. Un-deuterated and equilibrium-deuterated control samples are also prepared as previously described ${ }^{67}$. Frozen samples were later loaded onto a cryogenic autosampler ${ }^{68}$, thawed at $4{ }^{\circ} \mathrm{C}$, then passed over an immobilized pepsin column ( $16 \mu \mathrm{l}$ bed volume) for $30-40 \mathrm{sec}$ digestion. Proteolytic fragments were collected on a trap column and separated using Optimize Technologies $\mathrm{C} 18$ reverse phase analytical column (Halo EC-C18 $0.2 \times 50 \mathrm{~mm}, 2.7 \mu \mathrm{m}$ ) with an acetonitrile linear gradient (6.4\%-38.4\% over $30 \mathrm{~min}$ ). The effluent was directed into an OrbiTrap Elite Mass Spectrometer (ThermoFisher Scientific, San Jose, CA). Instrument settings were optimized to minimize the backexchange ${ }^{69}$. The data was acquired in either MS1 profile mode or data-dependent MS/MS mode. Peptide identification was done by the aid of Proteome Discoverer software (ThermoFisher). Mass envelope centroids of deuterated peptides were calculated with DXMS Explorer (Sierra Analytics Inc, Modesto, CA) then converted to corresponding deuterium incorporation with corrections for back-exchange $\mathrm{e}^{70}$.

\section{Site directed mutagenesis and enzymatic assay}

The fluorogenic peptide substrate Mca-Y-V-A-D-A-P-K(Dnp)-OH (R\&D Systems, Catalog \# ES007) was used to measure the activity of PreP. The reaction was monitored on a Synergy Neo microplate reader using an excitation wavelength of $320 \mathrm{~nm}$ and emission wavelength of $405 \mathrm{~nm}$. Reactions were carried out at $37^{\circ} \mathrm{C}$, using $5 \mathrm{nM}$ PreP with various concentrations of substrate $\mathrm{V}(5,10,20$, or $40 \mu \mathrm{M})$ in $200 \mu \mathrm{L}$ of 
buffer containing $20 \mathrm{mM}$ Tris, pH7.7, $150 \mathrm{mM} \mathrm{NaCl}, 1 \mathrm{mM} \beta$-mercaptoethanol. Degradation of substrate $\mathrm{V}$ was assessed by monitoring the fluorescence increase for $10 \mathrm{~min}$ at 30-second intervals. To calculate enzymatic activity, background subtraction and linear regression fitting were used to find the initial velocity, whereas specific activity was determined by comparing the maximal fluorescence converted from the known quantity of substrate $V$ by PreP.

\section{Cellular proliferation assay of human K562 leukemia cells}

MB-60 was purchased from MolPort. Leukemia K562 cell lines labelled with GFP (gifted from Luke Gilbert) were grown in RPMI-1640 medium with 10\% FBS, 0.05 mM 2-Mercaptoethanol and penicillin/streptomycin. Cells at $1 \times 10^{5}$ viable cells $/ \mathrm{mL}$ were added to 96 well plate with $100 \mu \mathrm{l} /$ well. MB60 was added to the indicated concentrations, $0.8 \mu \mathrm{M}$ to $25 \mu \mathrm{M}$. Cell growth was monitored continually every 4 hours up to 60 hours using IncuCyte S3s (Essen BioScience). The cell count from 12 hours to 40 hours was used to calculate their cell doubling time.

\section{Declarations}

\section{Author contributions:}

W.-J.T., W.G.L., S.L., B.C., C.S.P., and M.Z. designed the project. W.G.L. performed cryoEM grid preparation, data acquisition and processing assisted by H.W., M. P. and C.L. and overseen by W.-J. T., M. Z., B.C. and C.S.P. A.J.N. performed cryoET data acquisition and A.J.N., W.G.L. and J.M.M. performed the analysis. W.G.L., W.-J.T., and M.Z. built and refined cryoEM structural models. W.G.L. and S.M. performed protein purification and crystallographic data collection, built and refined structural models. W.G.L. performed protein purification for HDX-MS and D.L. and S.L. performed HDX-MS and analysis. W.G.L. purified proteins and performed SAXS studies. J. W. performed the inhibitor screen and characterization of MB-60 and J.W. and C.M.K. provided critical reagents for the crystallographic analysis. W.G.L, W.-J.T., J.V.L.K., J.M.M., and S.L. wrote the manuscript. S.M. J.W. M.Z., and B. C. contributed to manuscript finalization.

\section{Acknowledgement:}

We are grateful to Luke Gilbert for leukemia K562 cell line labelled with GFP and Srinivas Chakravarthy at BioCAT, APS for assisting with SAXS data collection and analysis. This work was supported by the NIH grant GM121964 to W.-J. Tang, GM103622 to Tom Irving at BioCAT, APS. Some of this work was performed at the Simons Electron Microscopy Center and National Resource for Automated Molecular Microscopy and National Center for CryoEM Access and Technology located at the New York Structural Biology Center, supported by grants from the Simons Foundation (SF349247) and the NIH National Institute of General Medical Sciences (GM103310, U24 GM129539). Use of the Advanced Photon Source was supported by the U.S. Department of Energy, Office of Basic Energy Sciences, under contract No. DEAC02-06CH11357. 


\section{References}

1. Eisner, V., Picard, M. \& Hajnoczky, G. Mitochondrial dynamics in adaptive and maladaptive cellular stress responses. Nat Cell Biol 20, 755-765 (2018).

2. Spinelli, J. B. \& Haigis, M. C. The multifaceted contributions of mitochondria to cellular metabolism. Nat Cell Biol 20, 745-754 (2018).

3. DiMauro, S. \& Schon, E. A. Mitochondrial disorders in the nervous system. Annu Rev Neurosci 31, 91-123 (2008).

4. Poveda-Huertes, D., Mulica, P. \& Vogtle, F. N. The versatility of the mitochondrial presequence processing machinery: cleavage, quality control and turnover. Cell Tissue Res 367, 73-81 (2017).

5. Pfanner, N., Warscheid, B. \& Wiedemann, N. Mitochondrial proteins: from biogenesis to functional networks. Nat Rev Mol Cell Bio 20, 267-284 (2019).

6. King, J. V. et al. Molecular Basis of Substrate Recognition and Degradation by Human Presequence Protease. Structure 22, 996-1007 (2014).

7. Johnson, K. A. et al. The closed structure of presequence protease PreP forms a unique $10,000 \AA^{3}$ chamber for proteolysis. EMBO J 25, 1977-1986 (2006).

8. Alikhani, N. et al. Decreased proteolytic activity of the mitochondrial amyloid-beta degrading enzyme, PreP peptidasome, in Alzheimer's disease brain mitochondria. J Alzheimers Dis 27, 75-87 (2011).

9. Fang, D. et al. Increased neuronal PreP activity reduces Abeta accumulation, attenuates neuroinflammation and improves mitochondrial and synaptic function in Alzheimer disease's mouse model. Hum Mol Genet 24, 5198-5210 (2015).

10. Brunetti, D. et al. Defective PITRM1 mitochondrial peptidase is associated with A amyloidotic neurodegeneration. Embo Mol Med 8, 176-190 (2016).

11. Langer, Y. et al. Mitochondrial PITRM1 peptidase loss-of-function in childhood cerebellar atrophy. $J$ Med Genet 55, 599-606 (2018).

12. Hipp, M. S., Kasturi, P. \& Hartl, F. U. The proteostasis network and its decline in ageing. Nat Rev Mol Cell Bio 20, 421-435 (2019).

13. Lopez-Otin, C., Blasco, M. A., Partridge, L., Serrano, M. \& Kroemer, G. The Hallmarks of Aging. Cell 153, 1194-1217 (2013).

14. Chiti, F. \& Dobson, C. M. Protein Misfolding, Amyloid Formation, and Human Disease: A Summary of Progress Over the Last Decade. Annual Review of Biochemistry, Vol 86 86, 27-68 (2017).

15. Greenwald, J. \& Riek, R. Biology of amyloid: structure, function, and regulation. Structure 18, 12441260 (2010).

16. Selkoe, D. J. \& Hardy, J. The amyloid hypothesis of Alzheimer's disease at 25 years. Embo Mol Med 8, 595-608 (2016).

17. Muller, U. C., Deller, T. \& Korte, M. Not just amyloid: physiological functions of the amyloid precursor protein family. Nat Rev Neurosci 18, 281-298 (2017). 
18. Sikanyika, N. L., Parkington, H. C., Smith, A. I. \& Kuruppu, S. Powering Amyloid Beta Degrading Enzymes: A Possible Therapy for Alzheimer's Disease. Neurochem Res 44, 1289-1296 (2019).

19. Lopez-Otin, C. \& Bond, J. S. Proteases: multifunctional enzymes in life and disease. J Biol Chem 283, 30433-30437 (2008).

20. Gillam, J. E. \& MacPhee, C. E. Modelling amyloid fibril formation kinetics: mechanisms of nucleation and growth. J Phys Condens Matter 25, 373101 (2013).

21. Morris, A. M., Watzky, M. A. \& Finke, R. G. Protein aggregation kinetics, mechanism, and curve-fitting: a review of the literature. Biochim Biophys Acta 1794, 375-397 (2009).

22. Malito, E. et al. Molecular bases for the recognition of short peptide substrates and cysteine-directed modifications of human insulin-degrading enzyme. Biochemistry 47, 12822-12834 (2008).

23. Danev, R., Yanagisawa, H. \& Kikkawa, M. Cryo-Electron Microscopy Methodology: Current Aspects and Future Directions. Trends Biochem Sci 44, 837-848 (2019).

24. Lyumkis, D. Challenges and opportunities in cryo-EM single-particle analysis. J Biol Chem 294, 5181-5197 (2019).

25. Murata, K. \& Wolf, M. Cryo-electron microscopy for structural analysis of dynamic biological macromolecules. Bba-Gen Subjects 1862, 324-334 (2018).

26. Ognjenovic, J., Grisshammer, R. \& Subramaniam, S. Frontiers in Cryo Electron Microscopy of Complex Macromolecular Assemblies. Annu Rev Biomed Eng 21, 395-415 (2019).

27. Chari, A. et al. ProteoPlex: stability optimization of macromolecular complexes by sparse-matrix screening of chemical space. Nat Methods 12, 859-+ (2015).

28. Noble, A. J. et al. Routine single particle cryoEM sample and grid characterization by tomography. Elife 7(2018).

29. Glaeser, R. M. Proteins, interfaces, and cryo-EM grids. Curr Opin Colloid In 34, 1-8 (2018).

30. Noble, A. J. et al. Reducing effects of particle adsorption to the air-water interface in cryo-EM. Nat Methods 15, 793-795 (2018).

31. Dandey, V. P. et al. Spotiton: A new method for vitrifying samples for cryoEM. Acta Crystallogr A 73, C1295-C1295 (2017).

32. Dandey, V. P. et al. Spotiton: New features and applications. J Struct Biol 202, 161-169 (2018).

33. Jain, T., Sheehan, P., Crum, J., Carragher, B. \& Potter, C. S. Spotiton: A prototype for an integrated inkjet dispense and vitrification system for cryo-TEM. J Struct Biol 179, 68-75 (2012).

34. Darrow, M. C., Moore, J. P., Walker, R. J., Doering, K. \& King, R. S. Chameleon: Next Generation Sample Preparation for CryoEM based on Spotiton. Microscopy and Microanalysis 25, 994-995 (2019).

35. Chow, K. M. et al. Mammalian pitrilysin: substrate specificity and mitochondrial targeting. Biochemistry 48, 2868-2877 (2009).

36. Zhang, Z. N. et al. Ensemble cryoEM elucidates the mechanism of insulin capture and degradation by human insulin degrading enzyme. Elife 7(2018). 
37. Chung, K. Y. et al. Conformational changes in the $\mathrm{G}$ protein $\mathrm{Gs}$ induced by the $\beta 2$ adrenergic receptor. Nature 477, 611-615 (2011).

38. Li, S. S. et al. Mechanism of intracellular cAMP sensor Epac2 activation: CAMP-induced conformational changes identified by amide hydrogen/deuterium exchange mass spectrometry (DXMS). The Journal of biological chemistry 286, 17889-17897 (2011).

39. Marciano, D. P., Dharmarajan, V. \& Griffin, P. R. HDX-MS guided drug discovery: small molecules and biopharmaceuticals. Current opinion in structural biology 28, 105-111 (2014).

40. Maianti, J. P. et al. Anti-diabetic activity of insulin-degrading enzyme inhibitors mediated by multiple hormones. Nature 511, 94-98 (2014).

41. Deprez-poulain, R. et al. Catalytic Site Inhibition of Insulin Degrading Enzyme by a Small Molecule Induces Glucose Intolerance in Mice. Nature Communications 6, 8250 (2015).

42. Wijaya, J. Utilizing Small Molecules to Study Mitochondrial Presequence-Degrading Protease PhD thesis, UCLA, (2015).

43. Horlbeck, M. A. et al. Mapping the Genetic Landscape of Human Cells. Cell 174, 953-+ (2018).

44. D'Imprima, E. et al. Protein denaturation at the air-water interface and how to prevent it. Elife 8(2019).

45. Ravelli, R. B. G. et al. Cryo-EM structures from sub-nl volumes using pin-printing and jet vitrification. Nat Commun 11, 2563 (2020).

46. Rubinstein, J. L. et al. Shake-it-off: a simple ultrasonic cryo-EM specimen-preparation device. Acta Crystallogr D Struct Biol 75, 1063-1070 (2019).

47. McCord, L. A. et al. Conformational states and recognition of amyloidogenic peptides of human insulin-degrading enzyme. Proceedings of the National Academy of Sciences of the United States of America 110, 13827-13832 (2013).

48. Tang, W. J. Targeting Insulin-Degrading Enzyme to Treat Type 2 Diabetes Mellitus. Trends Endocrinol Metab 27, 24-34 (2016).

49. Shen, Y., Joachimiak, A., Rosner, M. R. \& Tang, W. J. Structures of human insulin-degrading enzyme reveal a new substrate recognition mechanism. Nature 443, 870-874 (2006).

50. Cabrol, C. et al. Small-molecule activators of insulin-degrading enzyme discovered through highthroughput compound screening. PLoS One 4, e5274 (2009).

51. Maianti, J. P. et al. Substrate-selective inhibitors that reprogram the activity of insulin-degrading enzyme. Nat Chem Biol 15, 565-+ (2019).

52. Kukday, S. S. et al. Cell-permeable, small-molecule activators of the insulin-degrading enzyme. $J$ Biomol Screen 17, 1348-1361 (2012).

53. Suloway, C. et al. Automated molecular microscopy: the new Leginon system. J Struct Bio/151, 4160 (2005).

54. Zivanov, J. et al. New tools for automated high-resolution cryo-EM structure determination in RELION3. Elife 7(2018). 
55. Zheng, S., Polovcak, E., Armache, J. P., Cheng, Y. \& Agard, D. Anisotropic Correction of Beam-induced Motion for Improved Single-particle Electron Cryomicroscopy. Nat Methods 14, 331-332 (2017).

56. Zhang, K. Gctf: Real-time CTF determination and correction. J Struct Biol 193, 1-12 (2016).

57. Pettersen, E. F. et al. UCSF chimera - A visualization system for exploratory research and analysis. $J$ Comput Chem 25, 1605-1612 (2004).

58. Emsley, P., Lohkamp, B., Scott, W. G. \& Cowtan, K. Features and development of Coot. Acta Crystallogr D Biol Crystallogr 66, 486-501 (2010).

59. Liebschner, D. et al. Macromolecular structure determination using X-rays, neutrons and electrons: recent developments in Phenix. Acta Crystallogr D Struct Biol 75, 861-877 (2019).

60. Noble, A. J. \& Stagg, S. M. Automated batch fiducial-less tilt-series alignment in Appion using Protomo. J Struct Biol 192, 270-278 (2015).

61. Agulleiro, J. I. \& Fernandez, J. J. Tomo3D 2.0-exploitation of advanced vector extensions (AVX) for 3D reconstruction. J Struct Biol 189, 147-152 (2015).

62. Castano-Diez, D., Kudryashev, M., Arheit, M. \& Stahlberg, H. Dynamo: a flexible, user-friendly development tool for subtomogram averaging of cryo-EM data in high-performance computing environments. J Struct Biol 178, 139-151 (2012).

63. Minor, W., Cymborowski, M., Otwinowski, Z. \& Chruszcz, M. HKL-3000: the integration of data reduction and structure solution-from diffraction images to an initial model in minutes. Acta Crystallogr D Biol Crystallogr 62, 859-866 (2006).

64. Franke, D. et al. ATSAS 2.8: a comprehensive data analysis suite for small-angle scattering from macromolecular solutions. J Appl Crystallogr 50, 1212-1225 (2017).

65. Marsh, J. J. et al. Structural Insights into Fibrinogen Dynamics Using Amide Hydrogen/Deuterium Exchange Mass Spectrometry. Biochemistry 52, 5491-5502 (2013).

66. Coales, S. J. et al. Expansion of time window for mass spectrometric measurement of amide hydrogen/deuterium exchange reactions. Rapid communications in mass spectrometry: RCM 24, 3585-3592 (2010).

67. Xiao, K. et al. Revealing the architecture of protein complexes by an orthogonal approach combining HDXMS, CXMS, and disulfide trapping. Nat Protoc 13, 1403-1428 (2018).

68. Woods, V. L., Jr. \& Hamuro, Y. High resolution, high-throughput amide deuterium exchange-mass spectrometry (DXMS) determination of protein binding site structure and dynamics: utility in pharmaceutical design. Journal of cellular biochemistry. Supplement Suppl 37, 89-98 (2001).

69. Walters, B. T., Ricciuti, A., Mayne, L. \& Englander, S. W. Minimizing back exchange in the hydrogen exchange-mass spectrometry experiment. J Am Soc Mass Spectrom 23, 2132-2139 (2012).

70. Zhang, Z. \& Smith, D. L. Determination of amide hydrogen exchange by mass spectrometry: a new tool for protein structure elucidation. Protein Sci 2, 522-531 (1993).

\section{Tables}


Table 1

CryoEM data collection, refinement, and validation statistics.

\begin{tabular}{|c|c|c|c|c|c|}
\hline $\begin{array}{l}\text { Data collection and } \\
\text { processing }\end{array}$ & Apo-PreP & & & $A \beta$-bound PreP & $\begin{array}{l}\text { CS27- } \\
\text { bound } \\
\text { PreP }\end{array}$ \\
\hline Conformations & $\begin{array}{l}\text { partial Closed } \\
1(\mathrm{pC} 1)\end{array}$ & $\begin{array}{l}\text { partial Closed } \\
2(\mathrm{pC} 2)\end{array}$ & $\begin{array}{l}\text { Open } \\
\text { (0) }\end{array}$ & $\begin{array}{l}\text { partial } \\
\text { Closed } \\
\text { (pC) }\end{array}$ & $\begin{array}{l}\text { partial Closed } \\
(\mathrm{pC})\end{array}$ \\
\hline Magnification & 130,000 & & & 130,000 & 130,000 \\
\hline Voltage (kV) & 300 & & & 300 & 300 \\
\hline Total dose $\left(\mathrm{e}^{-} / \AA^{2}\right)$ & 62.36 & & & 66.39 & 66.14 \\
\hline Defocus range $(\mu \mathrm{m})$ & $-1.5--2.5$ & & & $-1.2--2.0$ & $-1.5--2.5$ \\
\hline Pixel size (Å) & 0.856 & & & 0.855 & 0.855 \\
\hline Symmetry imposed & $\mathrm{C} 1$ & & & $\mathrm{C} 1$ & $\mathrm{C} 1$ \\
\hline $\begin{array}{l}\text { Initial particle } \\
\text { images (no.) }\end{array}$ & 356,754 & 356,754 & 334,473 & 213,544 & $1,799,857$ \\
\hline $\begin{array}{l}\text { Final particle } \\
\text { images (no.) }\end{array}$ & 130,572 & 139,127 & 93,593 & 174,537 & 330,536 \\
\hline Map resolution $(\AA)$ & 3.7 & 3.9 & 4.0 & 3.3 & 4.6 \\
\hline FSC threshold & 0.143 & 0.143 & 0.143 & 0.143 & 0.143 \\
\hline EMDB & EMD-22278 & EMD-22279 & $\begin{array}{l}\text { EMD- } \\
22280\end{array}$ & $\begin{array}{l}\text { EMD- } \\
22281\end{array}$ & EMD-22282 \\
\hline \multicolumn{6}{|l|}{ Refinement } \\
\hline $\begin{array}{l}\text { Map sharpening } B \\
\text { factor }\left(\AA^{2}\right)\end{array}$ & -56 & -88 & -92 & -40 & -292 \\
\hline \multicolumn{6}{|l|}{ Model composition } \\
\hline Protein residues & 7704 & 7704 & 7697 & 7718 & 7711 \\
\hline Total atoms & 7704 & 7704 & 7697 & 7769 & 7711 \\
\hline Substrate & - & - & - & 51 & - \\
\hline \multicolumn{6}{|l|}{ B factors $\left(\AA^{2}\right)$} \\
\hline Protein & 111 & 132 & 102 & 90 & 136 \\
\hline Substrate & - & - & - & 88 & - \\
\hline R.m.s deviations & & & & & \\
\hline
\end{tabular}




\begin{tabular}{|llllll|}
\hline $\begin{array}{l}\text { Data collection and } \\
\text { processing }\end{array}$ & Apo-PreP & & & Aß-bound PreP & $\begin{array}{c}\text { CS27- } \\
\text { bound } \\
\text { PreP }\end{array}$ \\
\hline Bond length $(\AA)$ & 0.009 & 0.006 & 0.005 & 0.004 & 0.006 \\
\hline Bond angle $\left({ }^{\circ}\right)$ & 1.080 & 1.086 & 1.081 & 0.668 & 0.976 \\
\hline Ramachandran (\%) & & & & & \\
\hline Favored & 97.61 & 97.19 & 97.19 & 94.91 & 95.84 \\
\hline Allowed & 2.39 & 2.81 & 2.81 & 5.09 & 4.16 \\
\hline Outliers & 0.00 & 0.00 & 0.00 & 0.00 & 0.00 \\
\hline Validation & & & & & \\
\hline MolProbity score & 1.17 & 1.41 & 1.32 & 1.98 & 2.15 \\
\hline Poor rotamers $(\%)$ & 0.00 & 0.23 & 0.35 & 0.00 & 0.23 \\
\hline Clash score & 2.88 & 4.98 & 3.80 & 13.06 & 23.48 \\
\hline PDB & 6 XOS & 6 XOT & 6 XOU & 6 XOV & 6 XOW \\
\hline
\end{tabular}


Table 2

Data collection and structure refinement statistics

\section{MB-60-bound PreP}

\section{Data Collection}

Beamline

APS-19ID

Wavelength $(\AA)$

0.9792

Space group

C2

Cell dimension $(\AA)$

a

b

C

a

$\beta$

Y

Resolution $(\AA)$

$\mathrm{R}_{\text {meas }}(\%)^{\mathrm{a}}$

$R_{\text {p.i.m }}(\%)^{b}$

$\mathrm{CC}_{1 / 2}{ }^{\mathrm{C}}$

$\mathrm{CC}^{* \mathrm{~d}}$

1/sigma

Redundancy ${ }^{f}$

Completeness (\%)

Unique reflections

Refinement

$\mathrm{R}_{\text {work }}{ }^{\mathrm{g}}$

$R_{\text {free }}{ }^{h}$

No. atoms
245.6

85.5

158.2

90.0

127.5

90.0

$44.85-2.27$

$18.6(80.1)^{\mathrm{e}}$

$3.8(31.4)^{\mathrm{e}}$

$(0.793)^{\mathrm{e}}$

$(0.941)^{\mathrm{e}}$

$19.4(2.3)^{\mathrm{e}}$

$9.7(42.8)^{\mathrm{e}}$

$99.9(98.0)^{\mathrm{e}}$

119317

0.208 


\section{MB-60-bound PreP}

\section{Protein}

Water

B-factors

Protein

Substrate

Water

r.m.s. deviations

Bond lengths $(\AA)$

Bond angles $\left({ }^{\circ}\right)$

Ramachandran plot (\%)

Favorable region

Allowed region

Generously allowed region

Disallowed region

PDB code

${ }^{\mathrm{a}} R_{\text {meas }}=\Sigma_{\mathrm{hkl}}[\mathrm{n} /(\mathrm{n}-1)]^{1 / 2} \Sigma_{\mathrm{i}}\left|\mathrm{I}_{\mathrm{hkl}, \mathrm{l}}-<\mathrm{l}_{\mathrm{hkl}}>\right| / \Sigma_{\mathrm{hkl}}<\mathrm{I}_{\mathrm{hkl}}>$

${ }^{\mathrm{b}} R_{\text {p.i.m. }}=\Sigma_{\mathrm{hkl}}[1 /(\mathrm{n}-1)]^{1 / 2} \Sigma_{\mathrm{i}}\left|\mathrm{I}_{\mathrm{hkl}, \mathrm{I}}-<\mathrm{l}_{\mathrm{hkl}}>\right| / \Sigma_{\mathrm{hkl}}<\mathrm{I}_{\mathrm{hkl}}>$

${ }^{\mathrm{c}} \mathrm{CC}_{1 / 2}$ - Pearson correlation coefficient between random half-datasets - $\rho_{x^{\prime}, y}=\operatorname{cov}\left[(x, y) /\left(\sigma_{x} \sigma_{y}\right)\right]$

${ }^{\mathrm{d}} \mathrm{CC} *=\left[2 \mathrm{CC}_{1 / 2} /\left(1+\mathrm{CC}_{1 / 2}\right)\right]^{1 / 2}$

ethe outer resolution shell. Values in parentheses indicate the highest resolution shell

${ }^{\mathrm{f}} N_{\text {obs }} / N_{\text {unique }}$

${ }^{g} R_{\text {work }}=\Sigma_{h k l}|| \mathrm{F}_{\text {obs }}|-k| \mathrm{F}_{\text {calc }}|| / \Sigma_{h k l}\left|\mathrm{~F}_{\text {obs }}\right|$

${ }^{h} R_{\text {free, }}$ calculated the same as for $\mathbf{R}_{\text {work }}$ but on the $5 \%$ data excluded from the refinement calculation.

\section{Figures}


A
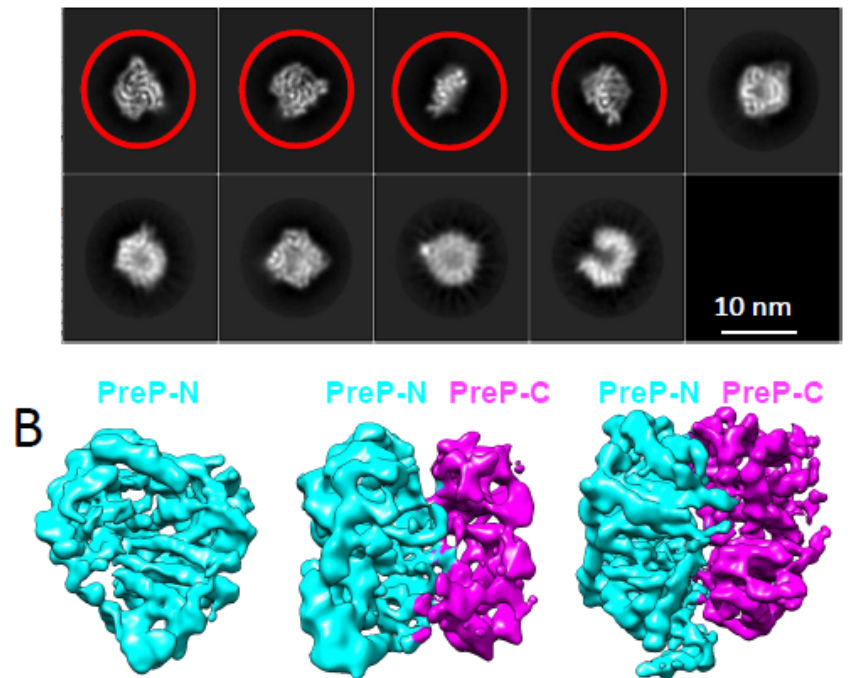

4.2 A, 327K

PreP-N only Missing PreP-C

C

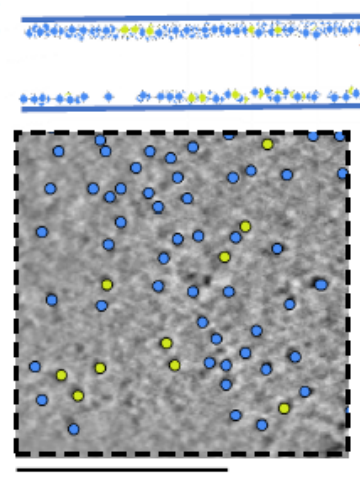

$100 \mathrm{~nm}$

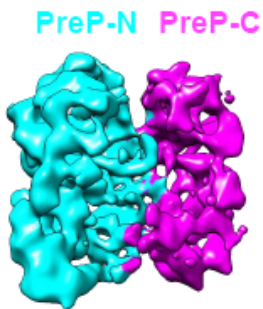

$5.3 \AA$, 34K

Open (O)

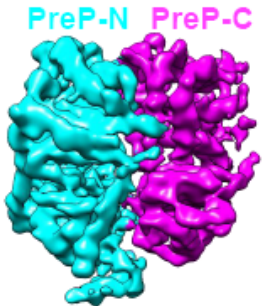

$4.5 \AA$, $50 \mathrm{~K}$ partial Open (po)
Missing some PreP-C

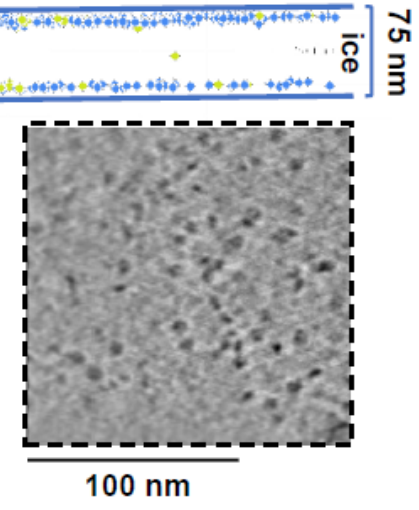

D Chameleon
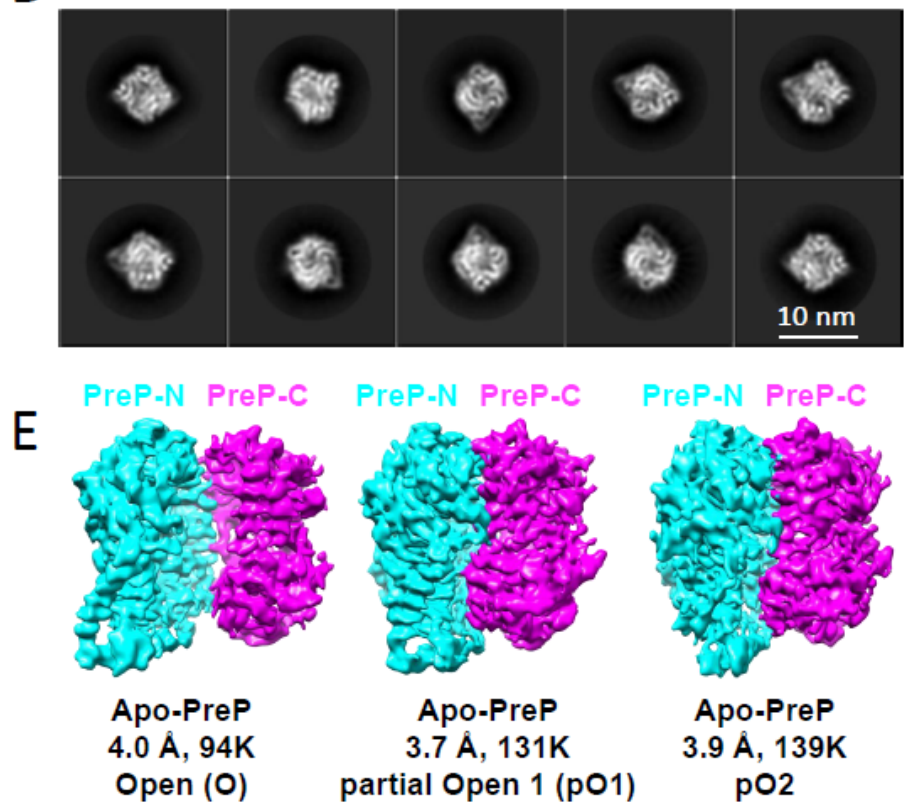

Apo-PreP

$3.7 \AA, 131 \mathrm{~K}$

partial Open 1 (po1)

F
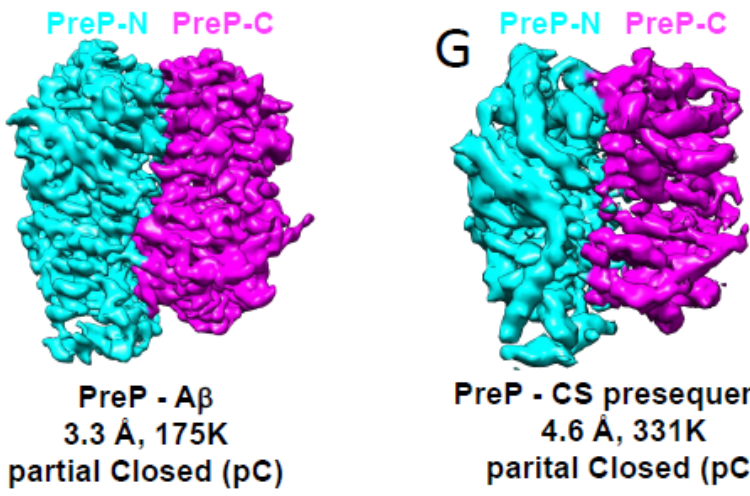

PreP - CS presequence

4.6 A, 331K parital Closed (pC)

\section{Figure 1}

CryoEM analysis of PreP. A) 2D classification, B) 3D classification, and C) cryoET analysis of PreP alone using grid prepared by Vitrobot. Top, Schematic diagrams of the average ice thickness (solid blue lines), and particles distribution in the ice. Almost all particles are on the air-water interface (770 particles), and only one full particle is not absorbed to the air-water interface. Bottom, Comparison of enlarged slice of tomograms with particles labels (left) and without particles labels (right). D) 2D and E) 3D classification of PreP alone using grid prepared by chameleon. 3D classification of PreP in complex with $A \beta(F)$ and citrate synthase presequence $(G)$. 

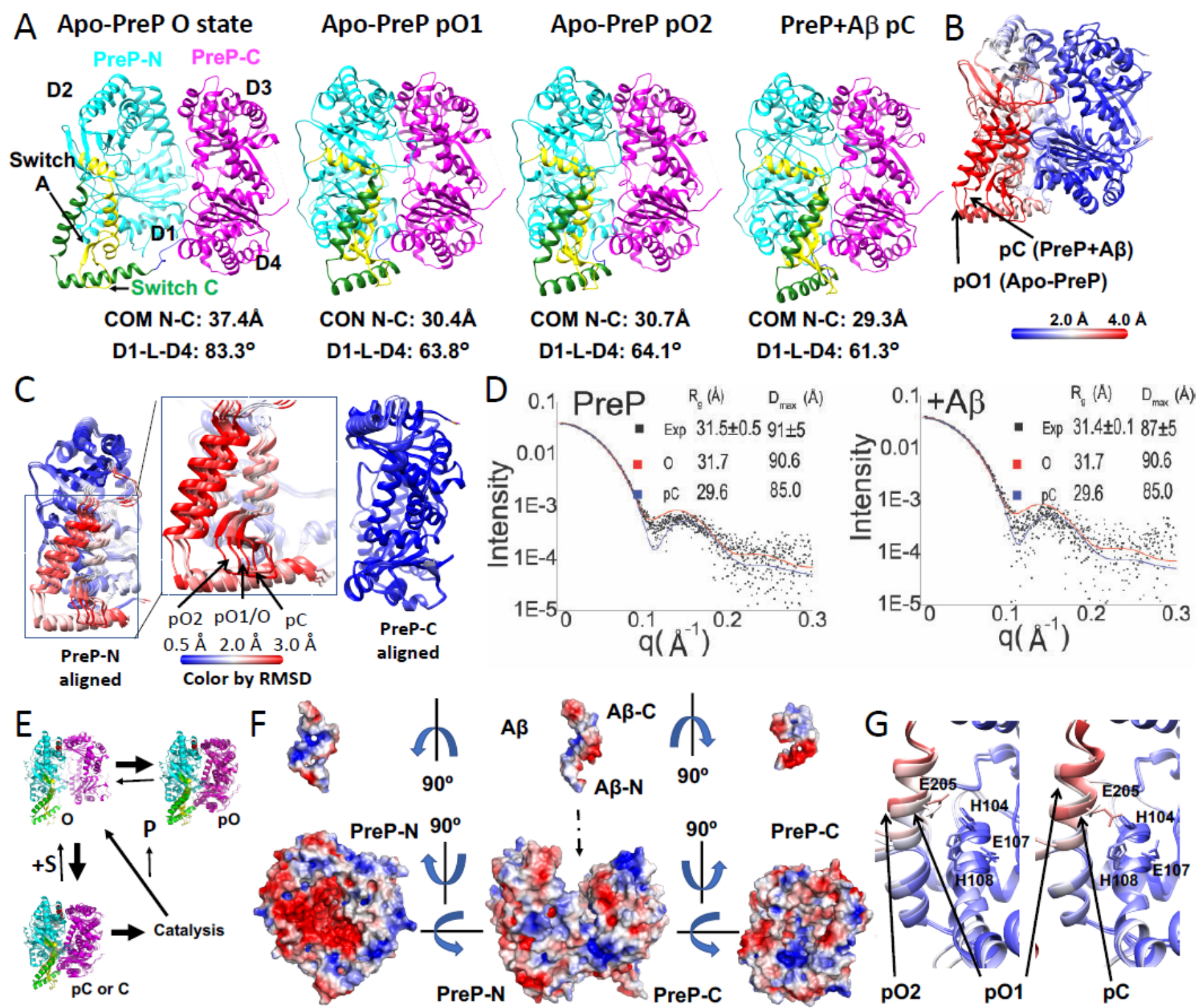

Figure 2

Comparison of PreP structures. (A) Four key cryoEM structures of PreP. Conformations of PreP open (0), partial open state 1 and 2 (p01 and p02), and AB-bound partial closed states (pC) are shown in ribbon. The distance between center of mass (COM) of PreP-N and PreP-C and the angle for COM of D1, aa 562564 at switch C, and D4 domain are listed below each conformation. (B) Alignment of p01 and pC state of PreP colored by RMSD. (C) Alignment of PreP-N and PreP-C of four distinct conformational states of PreP colored by RMSD. (D) SEC-SAXS analysis of PreP in the presence and absence of A 3 . (E) Model of PreP catalytic cycle. $S$ is substrate and $P$ is proteolytic products. (F) Structural basis of PreP open state primed to capture $A \beta$ by size and charge complementarity. (G) Structural comparison of the catalytic site of $\mathrm{pO}$ and $\mathrm{pC}$ states. 


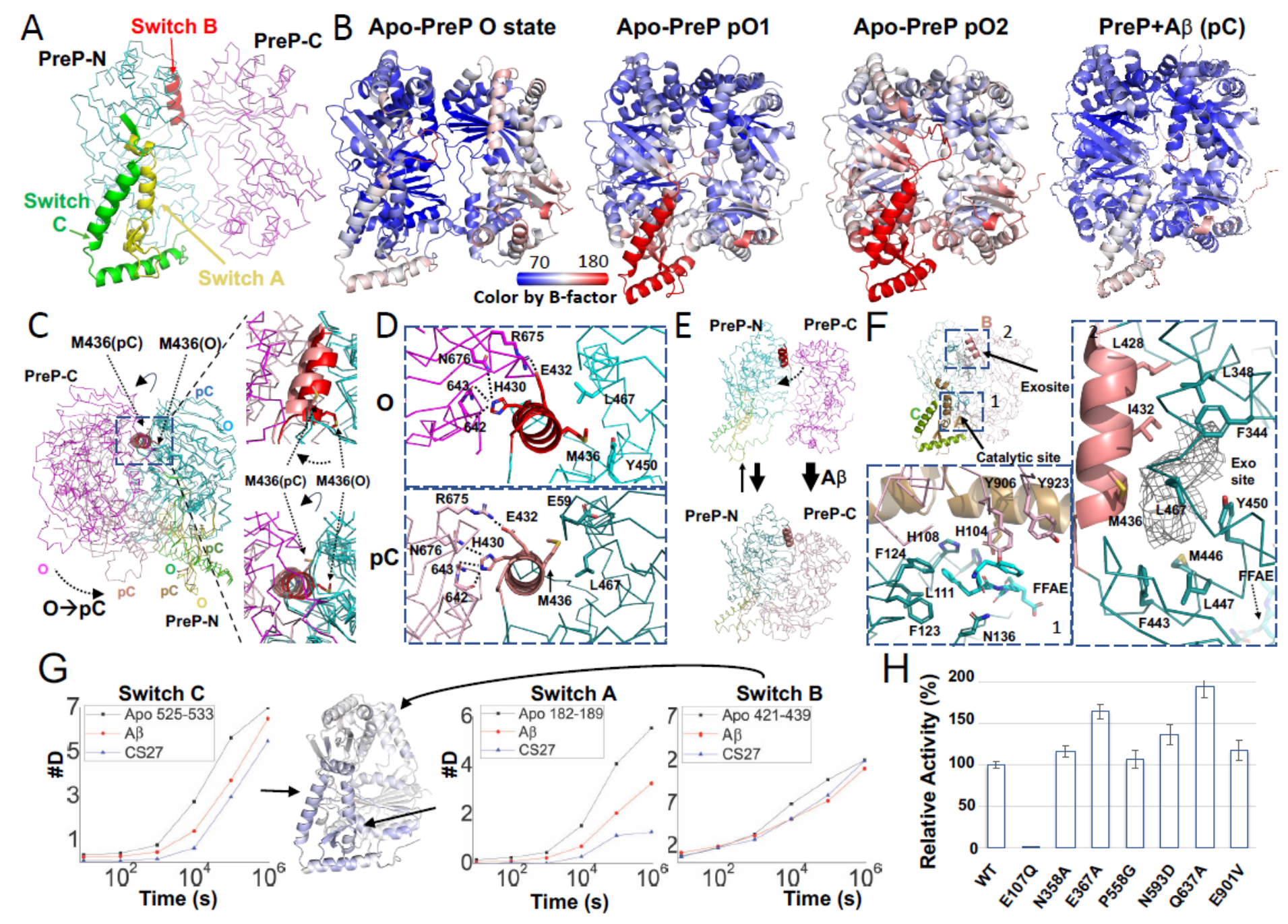

Figure 3

Conformational switch of PreP. (A) Three switch regions of PreP. (B) Four cryoEM PreP structures colored by B-factors. (C) Comparison of PreP open and partial closed state to show the rotation of switch $B$ helix that governs the open-closed transition. (D) The detailed interactions of switch B region in the open (top) and partial closed (bottom) states of PreP. (E) Model depicting how the rotation of switch B region governs the preferred parital closed state in the absence of substrate and the interaction of hydrophobic residues in the substrate, e.g., $A \beta$ induces the open to closed transition of PreP. (F) The interaction of $A \beta$ with the catalytic site (box 1) and exosite (box 2) of PreP. The key residues for $A \beta$ binding and those that form exosite are shown in stick and labeled. The density found in $A \beta$ bound PreP structure is shown in mesh. The 19FFAE22 in A $\beta$ is colored in cyan. (G) Differential HDX between PreP in the absence and presence of substrates, including $A \beta$ and citrate synthase presequence, CS27 mapped on the PreP-N structure. Differences in the average HDX are represented as percent change and colored with blue being slower exchange with CS27 and with red being faster. $(\mathrm{H})$ Relative catalytic activities of PreP mutants that have point mutations at residues residing at the interface between PreP-N and PreP-C. 

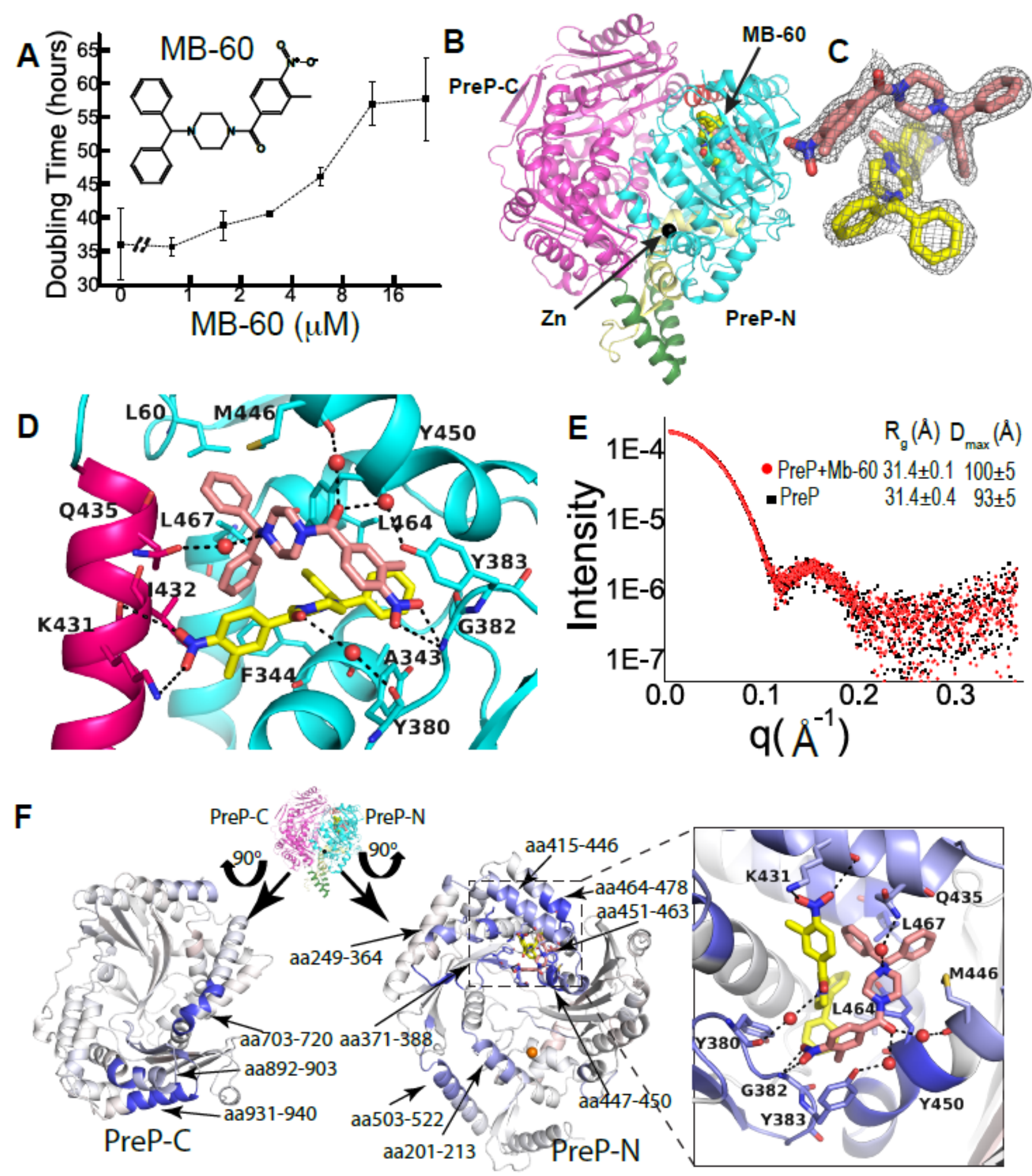

Figure 4

Structural analysis of PreP in complex with small molecule inhibitor, MB-60. (A) The effect of MB-60 on the doubling time of K562 Leukemia cells. The chemical formula of MB-60 is shown. (B) Overall structure of MB-60-bound PreP (PDB code: 4RPU) PreP is depicted in a ribbon representation. The carbons of two MB-60 molecules are colored in yellow and pink while $\mathrm{N}$ and $\mathrm{O}$ atoms are in blue and red, respectively. The catalytic zinc ion is in grey. C. 2 mFo-DFc omit map of MB-60 to depict the closed contact between 
two MB-60 molecules at the exosite. The map was contoured to 1б. (D) Detailed interactions of two MB60 with PreP side chains within the exosite. (E) SEC-SAXS scattering profile of PreP in the presence or absence of MB-60 (dotted lines). Theoretical scattering profiles of open and closed PreP (solid lines) were modeled and calculated by CRYSOL. (F) Differential HDX between PreP in the absence and presence of MB-60 mapped on the MB-60 bound PreP crystal structure. Differences in the average HDX are represented as percent change and colored with blue being slower exchange with MB-60 while with red being faster.

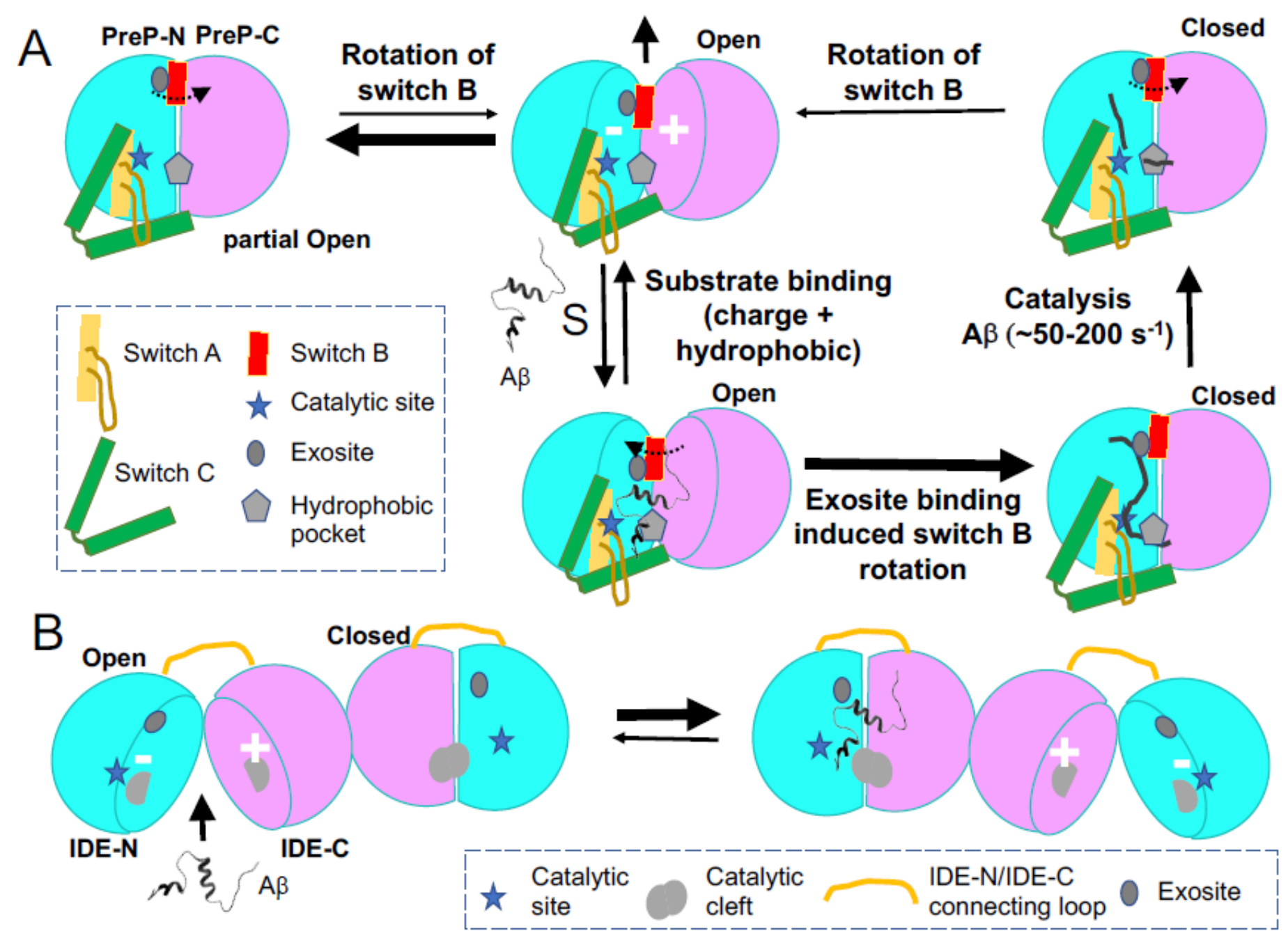

\section{Figure 5}

Comparison of key structural features of PreP and IDE for catalysis. (A) A model of the catalytic cycle of PreP to depict the structural basis for the conformational switch and substrate recognition. (B) A model of dimeric IDE to depict the allosteric regulation. The domains and switch regions are depicted as the cartoon and colored the same as figures above. The detailed description is in the discussion.

\section{Supplementary Files}

This is a list of supplementary files associated with this preprint. Click to download. 
- Movie1.avi

- Movie2.mp4

- Movie3.mp4

- Movie4.mp4

- Movie5.mp4

- SupplementalFigures.pdf 